\title{
Adrenomedullin serves a role in the humoral pathway of delayed remote ischemic preconditioning via a hypoxia-inducible factor-1 $\alpha$-associated mechanism
}

\author{
WENPEI DONG ${ }^{1,2}$, PING YU ${ }^{2}$, TIELONG ZHANG ${ }^{1,2}$, CHENZHUANG ZHU $^{1,2}$, JUN QI ${ }^{1,2}$ and JUNHAO LIANG ${ }^{1,2}$ \\ ${ }^{1}$ Department of Urology, Xinhua Hospital, School of Medicine, Shanghai Jiao Tong University, \\ Shanghai 200092; ${ }^{2}$ School of Medicine, Shanghai Jiao Tong University, Shanghai 200025, P.R. China
}

Received July 13, 2017; Accepted January 12, 2018

DOI: $10.3892 / \mathrm{mmr} .2018 .8450$

\begin{abstract}
Remote ischemic preconditioning (RIPC) is a minimally invasive method that provides protection by reducing injury to the heart, kidneys, brain and other tissues or organs. RIPC may improve the outcome in patients undergoing surgery. Although the role of RIPC has been studied, the results remain controversial. It is difficult to confirm whether RIPC has a kidney protective effect and the understanding of the preconditioning signal pathway involved remains unclear. In the present study, the effect of RIPC in urology was evaluated. The protection against renal damage was assessed by investigating the potential mediator, hypoxia-inducible factor-1 $\alpha$ (HIF-1 $\alpha)$, and the functional adrenomedullin (ADM) pathway. Male Sprague-Dawley (SD) rats were used in the present study. The animal model of kidney damage induced by ischemia reperfusion (IR) was used to investigate the protective effect of the acute and delayed phase RIPC. Furthermore, the protective effects of RIPC mediated by a HIF-1 $\alpha$-ADM pathway were assessed. The indexes of renal function and oxidative damage indicators were measured by $\mathrm{Cr}$, BUN, mALB, $\beta 2-\mathrm{MG}, \mathrm{MPO}$, MDA and SOD assays, and the expression of HIF-1 $\alpha$ and ADM were detected by western blot analysis, immunohistochemistry and ELISA assays. Tubular score, determined using hematoxylin and eosin staining, was used to evaluate renal tissue damage. Applying RIPC prevented IR-induced renal dysfunction and oxidative damage by decreasing $\mathrm{Cr}$, BUN, mALB, $\beta 2-M G$, MPO, MDA levels and increasing SOD activity. Findings showed that delayed RIPC had an improved effect compared with acute treatment. Delayed RIPC also upregulated the expression of HIF-1 $\alpha$ and $\mathrm{ADM}$, indicating that the protective effect of the delayed RIPC may be associated
\end{abstract}

Correspondence to: Professor Junhao Liang, Department of Urology, Xinhua Hospital, School of Medicine, Shanghai Jiao Tong University, 1665 Kongjiang Road, Shanghai 200092, P.R. China E-mail: liangjunhao@xinhuamed.com.cn

Key words: remote ischemic preconditioning, hypoxia-inducible factor-1 $\alpha$, adrenomedullin, ischemia reperfusion with a HIF-1 $\alpha$-ADM-mediated mechanism. The effect of the delayed RIPC to reduce IR-induced renal damage and increase ADM expression was enhanced by HIF-1 $\alpha$ agonists DMOG and BAY 85-3934, whereas the effect was whittled by HIF-1 $\alpha$ antagonists YC-1 and 2-MeOE2. Furthermore, receiving ADM also offered protection to the kidney in comparison with the IR+Vehicle group. These findings suggest that RIPC prevents IR-mediated renal damage by HIF-1 $\alpha$ via an ADM humoral pathway. In the present study, RIPC provided an effective renal protection. ADM could also offer protection regulated by HIF-1 $\alpha$ in renal tissue. However, the mechanism of ADM as a protective factor in RIPC requires further research.

\section{Introduction}

The kidney, an organ particularly prone to ischemic injury, will undergo a period of ischemia during some urologic surgeries such as renal partial nephrectomy or kidney transplantation. Ischemic injury leads to necrosis of tubular epithelial cells and acute renal failure, which is a major cause of morbidity and mortality. Ischemia-reperfusion injury (IRI) led to inevitable renal damage. Thus, reducing the damage to ischemic tissues has been an important therapeutic goal. Various potentially attractive measures to achieve this goal have been discovered and applied to protect organs from IRI. In 1986, the phenomenon of ischemic preconditioning (IPC) which could obviously reduce myocardial infarct size, was discovered (1). In 1993, an update version-remote ischemic preconditioning (RIPC)-was initially described by Przyklenk et al that decreased infarct area (2). Considering these reviews, using RIPC as a less invasive method, brief cycles of limb ischemia and reperfusion as a remote precondition stimulus achieved by applying a simple tourniquet around the lower or upper limb was a noninvasive and comparatively inexpensive procedure $(3,4)$. However, results of RIPC were controversial $(5,6)$. Results from animal experiments and clinical trials indicated that RIPC might offer neuroprotective effects by regulating multiple cellular processes (3). RIPC was previously shown to have a protective effect from renal IRI in rats preexposed to chronic hypoxia via hypoxia-inducible factor- $1 \alpha$ (HIF-1 $\alpha$ ). RIPC is extensively documented as a method of protecting organs, including the kidney, from IRI. 
Currently, the mechanisms of RIPC have been considered to involve pathways, comparatively similar to IPC: (1) Neuronal pathway, (2) systemic response, (3) humoral pathway $(7,8)$. The neuronal and systemic response pathway have been studied in depth whereas the humoral factor that conveyed the preconditioning signal from the remote to the target has not yet been identified. The effective endogenous factors currently confirmed are adenosine (9), bradykinin (10), opioids (11), and calcitonin gene-related peptide (12), which is released from the remote organ or tissue during the preconditioning treatment and conveyed to the target organ through the bloodstream.

Furthermore, the transcription factor HIF-1 $\alpha$ has recently been revealed to be a potential mediator of RIPC-induced protection (13). Evidence indicates that HIF-1 $\alpha$ played an important role in the kidney and other organs involved in mediating protection. HIF-1 $\alpha$ activation offered obvious protection to the kidney from IRI for the survival of renal epithelial cells.

The protective effects of HIF-1 $\alpha$ activation in renal IRI might be mediated by a number of different pathways. Studies have shown that heme oxygenase-1 and erythropoietin are involved in this process (14). However, the exact humoral candidate remained unknown; it appears that the protective factor is thermolabile, hydrophobic and has a molecular mass range from 3.5 to $8 \mathrm{kDa}(15,16)$.

Adrenomedullin (ADM), a biologically active peptide, was first isolated from human pheochromocytoma tissue extracts, and later affirmed to be present extensively in vascular endothelium, heart, lung and kidney (17). It had an important effect on heart and vascular protection, angiogenesis, central nervous system protection, tumor growth-promoting action, anti-inflammation, receptors, and intracellular signalling system (18). In addition, it was regulated by HIF-1 $\alpha$ and its molecular mass was approximately $5 \mathrm{kDa}$ in the humoral candidate range $(19,20)$.

Thus, the purpose of this study was to investigate whether RIPC offered protection to kidneys with ischemia reperfusion injury and to determine whether ADM was the humoral candidate in RIPC based on the former result.

\section{Materials and methods}

Animals. In accordance with principles and guidelines of the Guide for the Care and Use of Laboratory Animals (US National Institutes of Health Publication), all experiments were performed in the laboratory of the Department of Pharmacology at Shanghai Jiao Tong University School of Medicine. Male SD rats weighing 210 \pm 20 g were given standard rat chow with free access to water and fasted, but allowed water ad libitum $12 \mathrm{~h}$ prior to experimentation. The present study was approved by the Ethics committee of Xinhua Hospital affiliated to Shanghai Jiao Tong University School of Medicine (Shanghai, China).

There are two phases of the study: Part 1, a comparing phase, in which we applied RIPC in a rat model and compared whether RIPC offered renal protection; and part 2, an intervention phase, in which we used drugs such as agonists and antagonists to confirm our assumption of the humoral candidate in RIPC.

Part 1: Twenty-four rats were randomised into four groups ( $n=6$ per group): Sham group, ischemia/reperfusion (IR) group, acute phase RIPC group, and delayed phase RIPC group. The sham group had sham surgery. The IR group underwent resection surgery of the right kidney and a microvascular clamp was placed on the left renal pedicle for $45 \mathrm{~min}$, and then reperfusion resumed (21). After $30 \mathrm{~min}$ of RIPC (22), the acute RIPC and delayed RIPC groups underwent the same step as the IR group. The difference between the acute and delayed groups was that the delayed group underwent IR surgery after a 24-hour period. All animals were sacrificed for specimen collection $24 \mathrm{~h}$ after surgery.

Part 2: We compared two phases of RIPC and acquired the better RIPC protocol in part 1. In part 2, 48 rats were randomly divided into eight groups based on the results in part 1 and the following drugs were used ( $\mathrm{n}=6$ per group): Sham+Vehicle $(21,23)$, IR+Vehicle (21), Delayed+Vehicle, Delayed+YC-1 (2 mg/kg) (23), Delayed+2-Methoxyestradiol (2-MeOE2) (20 mg/kg) (24), Delayed+DMOG (40 mg/kg) (25), Delayed+Molidustat (BAY 85-3934) (2.5 mg/kg) (26), IR+ADM (12 $\mu \mathrm{g} / \mathrm{kg})(27)$. All the injections were given intraperitoneally $2 \mathrm{~h}$ before the time of RIPC with an injection volume of $0.5 \mathrm{ml}$. The RIPC protocol adopted was on the basis of part $1(4,22)$. YC-1 and 2-MeOE2 were agents that inhibited HIF-1 activity in vivo and in vitro $(24,28)$. As a result, YC-1 could also decrease the expression level of the HIF-1 inducible genes, including ADM (29,30). DMOG and BAY 85-3934, known as the prolyl hydroxylase (PHD) inhibitor, slowed the degradation of the HIF-1 $\alpha$, relatively increasing the expression of HIF-1 $(26,31)$.

Biological samples and tissue handling. Blood was obtained from the renal artery before right nephrectomy and left kidney harvest. Urine was collected in $24 \mathrm{~h}$ after surgery. The left kidney was harvested for biochemical analysis, and immunohistochemical and histopathological use. The levels of urine microalbumin (mALB), serum beta2-microglobulin $(\beta 2-\mathrm{MG})$, creatinine $(\mathrm{Cr})$, blood urea nitrogen (BUN), together with superoxide dismutase (SOD), malondialdehyde (MDA), and myeloperoxidase (MPO), the three of which originated from kidney tissue were tested by commercial assay kits (Jiancheng Bioengineering Institute, Nanjing, China). ADM in blood and kidney tissue was detected by an ELISA kit (Phoenix Pharmaceuticals, Inc., Burlingame, CA, USA). Western blot analyses were conducted by using HIF- $1 \alpha$ antibody (1:500; Novus Biologicals, Littleton, CO, USA). To detect HIF-1 $\alpha$ and ADM expression, immunohistochemical staining was performed by the use of HIF-1 $\alpha$ (1:50; Novus Biologicals), ADM (1:200; Santa Cruz Biotechnology, Inc., Dallas, TX, USA) antibodies. The histopathological findings on the kidney tissue stained with hematoxylin and eosin were measured by the tubular score method $(32,33)$.

Data analysis. Data were given as mean \pm standard deviation. Software used for statistical analysis was Graphpad Prism ${ }^{\circledR}$ (v5.01 for Windows; GraphPad Software, Inc., La Jolla, CA, USA) and IBM SPSS Statistics (v19.0 for Windows; IBM Corp., Armonk, NY, USA). All data were tested using a one-way analysis of variance with the Tukey multiple comparison test. $\mathrm{P}<0.05$ was considered to indicate a statistically significant difference. 


\section{Results}

Prevention of IR induced renal dysfunction and oxidative damage by RIPC. A significant increase in levels of $\mathrm{Cr}, \mathrm{BUN}$, mALB, $\beta 2-\mathrm{MG}, \mathrm{MPO}, \mathrm{MDA}$ and decreased activity of SOD were shown in the IR group, indicating an obvious renal dysfunction and oxidative damage. Treatment with acute and delayed RIPC significantly decreased the renal damage with a lower level of $\mathrm{Cr}$, BUN, mALB, $\beta 2-\mathrm{MG}, \mathrm{MPO}, \mathrm{MDA}$ and increased level of SOD compared with the IR group. In addition, the indexes of renal dysfunction and oxidative damage indicators induced by IR were reversed more significantly in the delayed RIPC, which seemed to have a better protective effect than the acute phase in part 1 as outlined in Table $\mathrm{I}(\mathrm{P}<0.05)$. These results strongly suggested that delayed RIPC produced marked beneficial profiles against IR-induced renal injury.

Modulated effects of RIPC on the renal ischemic injury related to $H I F-1 \alpha$. Effects of RIPC on the renal ischemic injury related to HIF-1 $\alpha$ were assessed (Fig. 1). Western blot analysis and immunohistochemistry staining of renal tissue showed an increase in HIF-1 $\alpha$ expression of renal tissue in acute and delayed RIPC groups in comparison with the IR group, and the level of HIF-1 $\alpha$ expression in the delayed RIPC group was higher than in the acute group (Fig. 1A, B, D, E; $\mathrm{P}<0.05)$. RIPC significantly increased the activity of SOD and decreased the production of MPO and MDA in the two RIPC groups compared with the IR group. It was also found that SOD in the delayed RIPC group was higher than in the acute group as outlined in Table I $(\mathrm{P}<0.05)$. Interestingly, the blood levels of ADM were increased in the delayed RIPC group, whereas there was no significant change in the acute group (Fig. 1C). What's more, the expression of ADM in delayed RIPC group was also higher than the acute one (Fig. 1F, G). In addition, the histopathological results and tubular score also These results suggested a better protective effect of delayed rather than acute RIPC (Fig. 1H, I).

Based on these results, delayed RIPC was chosen as the effective measure to prevent renal damage in the following experiments as Delayed+Vehicle group. By pre-treating with YC-1 and 2-MeOE2, both HIF-1 $\alpha$ antagonists, the expression of HIF-1 $\alpha$ was significantly downregulated in comparison with the Delayed+Vehicle group. DMOG and BAY 85-3934 pretreatment could markedly upregulate HIF-1 $\alpha$ expression in the Delayed+DMOG and Delayed+BAY 85-3934 group than in the Delayed+Vehicle group (Fig. 2; $\mathrm{P}<0.05$ ). In the Delayed+ YC-1 and Delayed+2-MeOE2 group, the renal injury was obviously accentuated after YC-1 and 2-MeOE2 pretreatment with a higher tubular score, increased levels of $\mathrm{Cr}$, BUN, mALB, ß2-MG, MPO, and MDA, and a marked decline in SOD activity that almost counteracted the RIPC protection (Table I). However, the protective effect of RIPC could be dramatically augmented by DMOG in the Delayed+DMOG group and BAY 85-3934 in the Delayed+BAY 85-3934 group with slighter renal injury than in the Delayed+Vehicle group by decreasing tubular score and production of $\mathrm{Cr}$, MPO, and MDA (Table I, Fig. 3A, B).

Protective effects of RIPC against IR induced renal damage mediated by $A D M$. Immunochemistry staining and ELISA detection of renal tissue revealed that RIPC could markedly

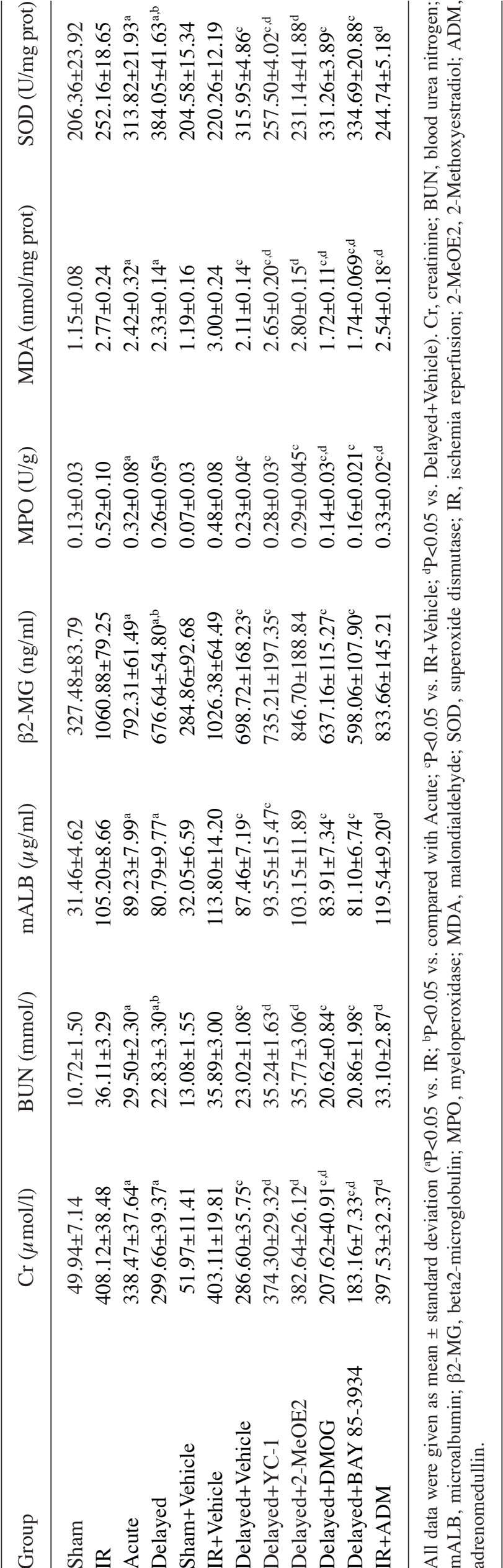


A

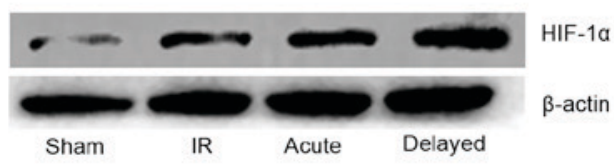

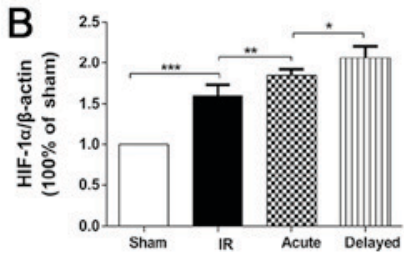

D

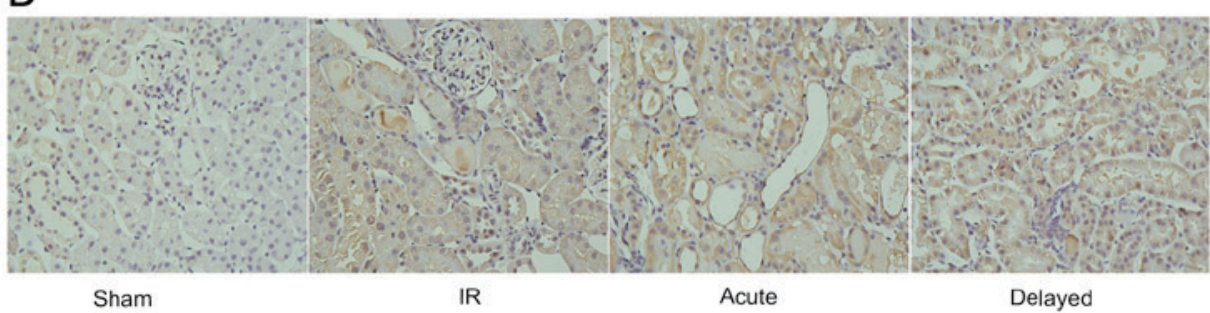

F

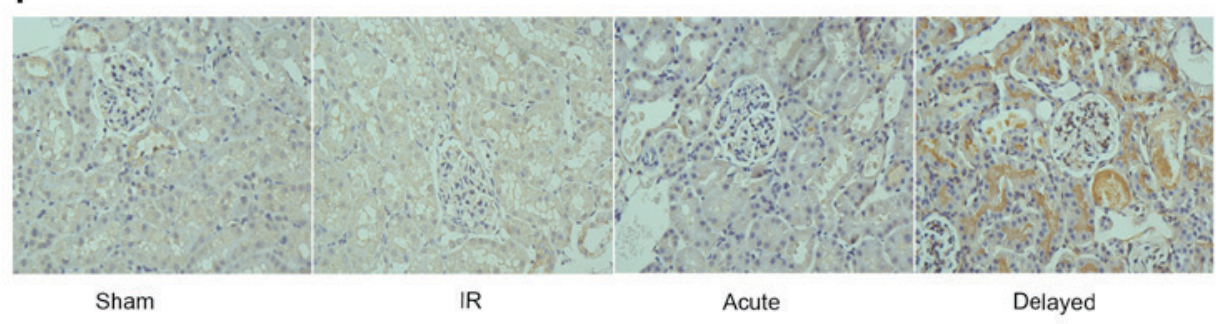

H

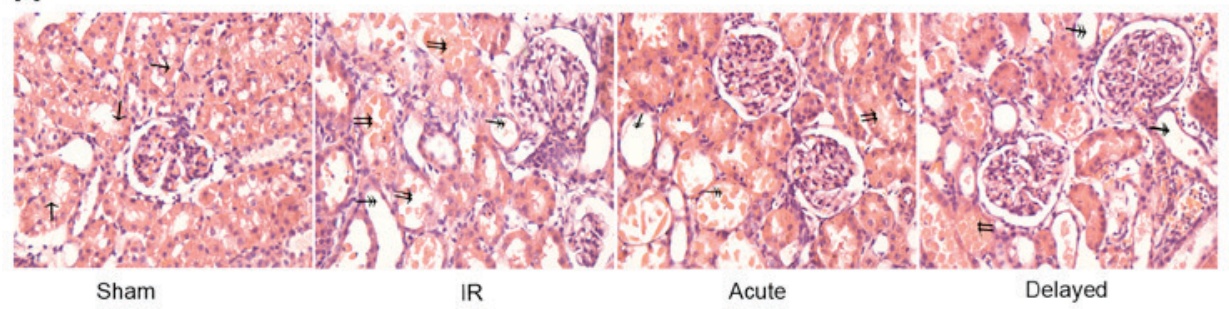

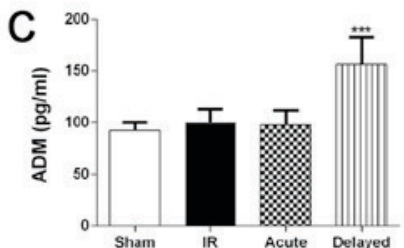

E

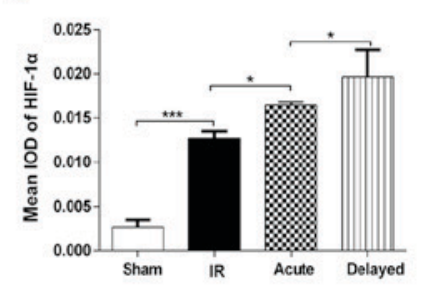

G

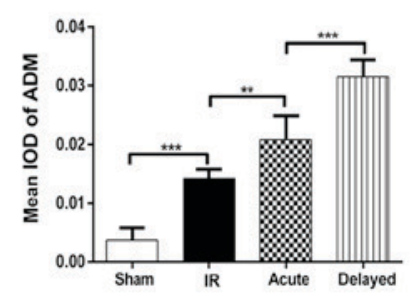

I

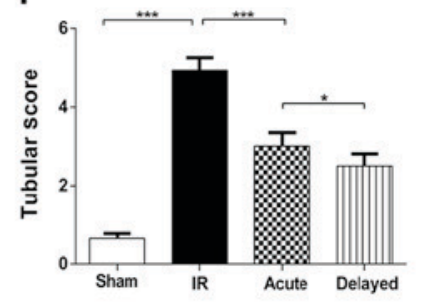

Figure 1. RIPC influenced the expression of HIF-1 $\alpha, \mathrm{ADM}$, and tubular score (IHC and HE; magnification, $\mathrm{x} 400)\left({ }^{*} \mathrm{P}<0.05 ;{ }^{* *} \mathrm{P}<0.01 ;{ }^{* * *} \mathrm{P}<0.001 ; \uparrow\right.$ tubular epithelial cell flattening, brush border loss, and apoptosis; $\uparrow$ lumen flat expansion and cast; $\uparrow$ tubular lumen multifocal necrosis and debris). (A and B) The expression of HIF-1 $\alpha$ detected by Western blot. (C) The level of ADM in blood. (D and E) The immunohistochemistry staining of HIF-1 $\alpha$ in renal tissue. (F and G) The immunohistochemistry staining of ADM. (H) Hematoxylin and eosin staining of renal tissue. (I) The tubular score of renal injury.

upregulate ADM expression, accompanied by HIF-1 $\alpha$, in the kidney. After receiving exogenous ADM, the renal tissues of the IR+ADM group showed notably increased ADM expression but not in the blood compared with the IR+Vehicle group (Fig. 2E-H). Treatment with ADM also led to increased activity of SOD, lowering the levels of MPO and MDA and improving tubular score for renal histopathological changes (Table I, Fig. 3A, B). All of these results revealed that ADM offered slight protection to the kidney than did the IR+Vehicle group, indicating this humoral factor was involved in the process of RIPC's protection against IR-induced renal injury. In addition, drug intervention targeting HIF-1 also regulated the expression of ADM. Pretreatment with YC-1, the antagonist of HIF-1, in the Delayed+YC-1 group visibly downregulated the production of ADM with accelerated renal damage. So did the Delayed+2-MeOE2 group. However, DMOG and BAY 85-3934, the agonist of HIF-1, significantly increased the expression of ADM (Fig. 2E, F, G, H) and improved renal function (Table I). The histopathological findings in (Fig. 3A, B) showed a few of tubular epithelial cell flattening, brush border loss, and apoptosis were observed in the Sham+Vehicle group. Consequently, the Sham+Vehicle group got the lowest score. However, the IR+Vehicle group which scored the highest, in addition to the histopathological changes mentioned for the Sham+Vehicle group, had the pathological changes of tubular lumen multifocal necrosis and debris, lumen flat expansion and cast. In the Delayed+Vehicle, Delayed+YC-1, Delayed+2-MeOE2, Delayed+DMOG, Delayed+BAY 85-3934 groups, the lumen necrosis and flat expansion and cast were also observed, but the damage and score was less than the IR+Vehicle group. All these results indicated that ADM might act as a downstream humoral factor regulated by HIF-1 $\alpha$. The relationship between these results and the complete mechanism needs further investigation.

In summary, we found RIPC could offer a protective effect against IR-induced renal injury by reversing the renal dysfunction and oxidative damage indicators in this study. Results also showed that the effect of RIPC was mediated by HIF- $1 \alpha$ and a downstream humoral factor ADM. However, molecular mechanism is still unclear and further investigation is needed. 
A

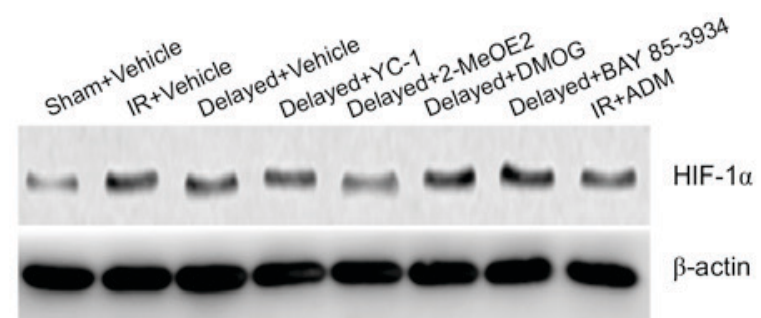

C

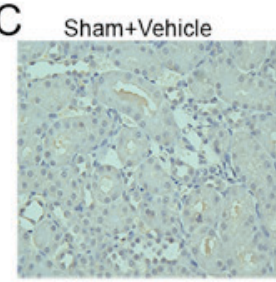

Delayed+2-MeOE2
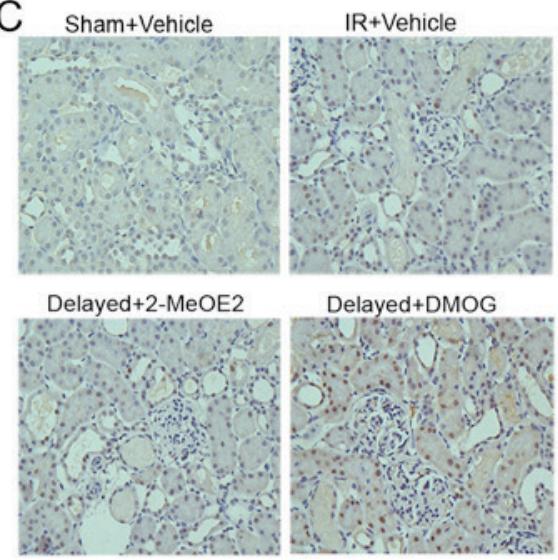

Delayed+Vehicle

Delayed+DMOG
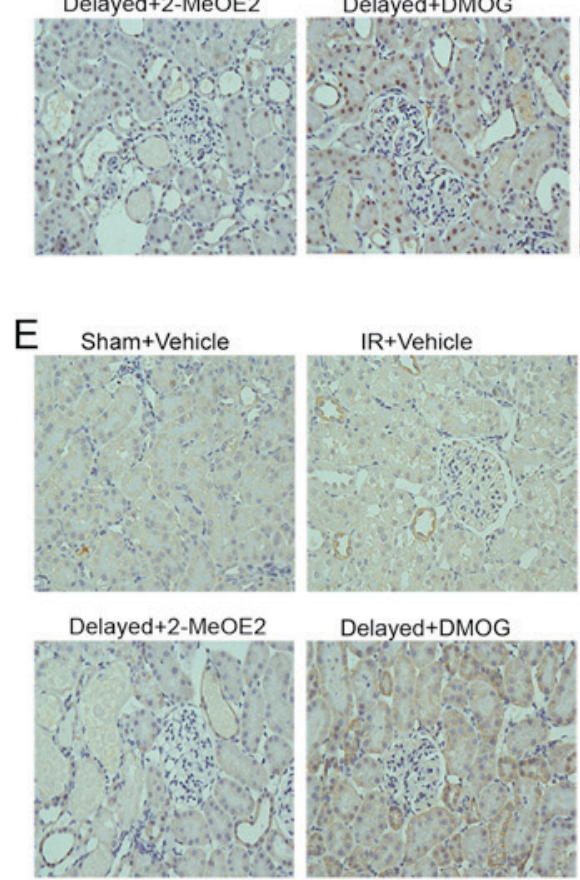

G

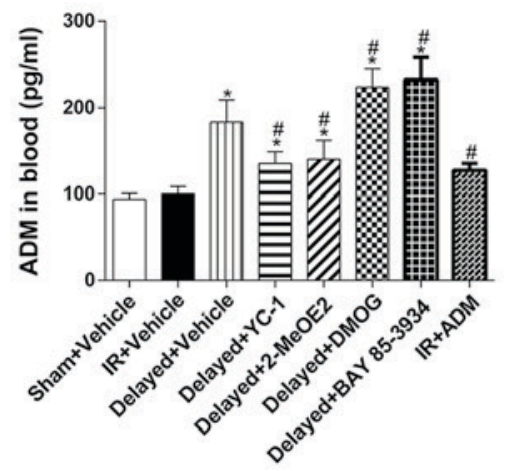

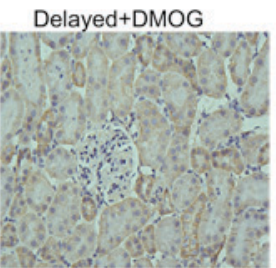

IR+Vehicle
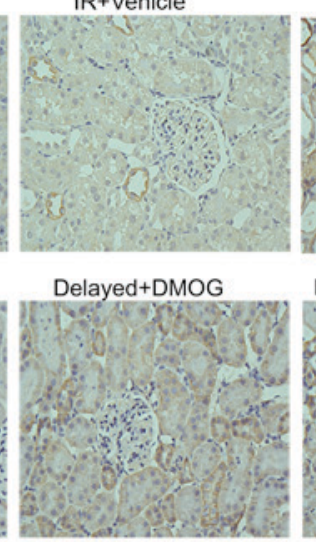

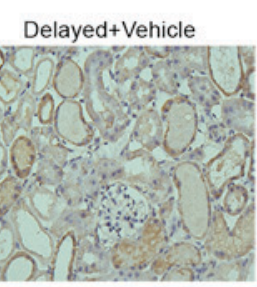

Delayed+BAY 85-3934

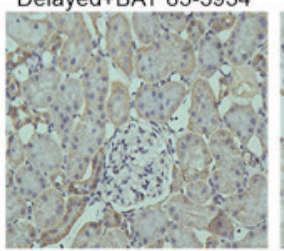

Delayed + YC-1

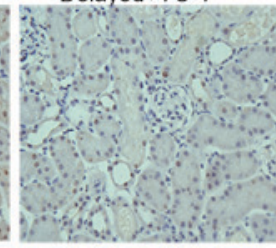

IR+ADM

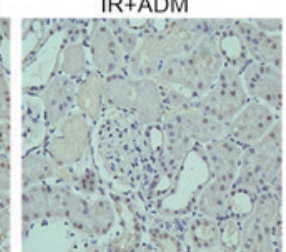

Delayed + YC-1

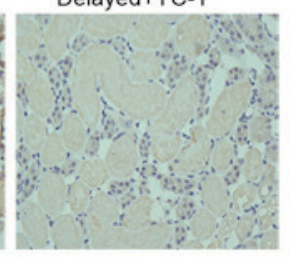

IR+ADM

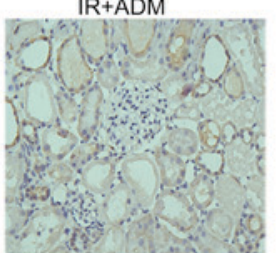

B

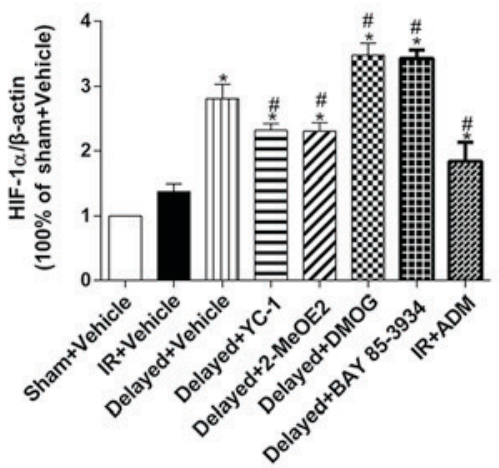

D

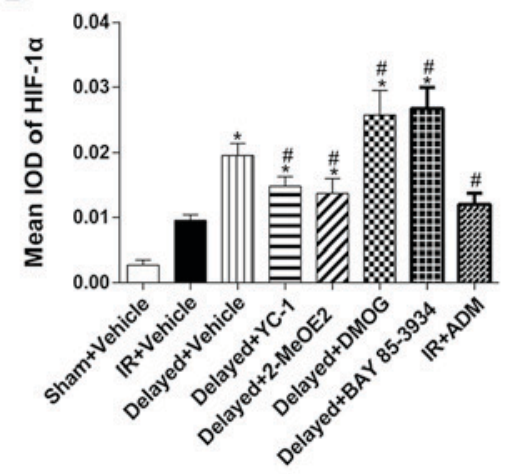

$\mathrm{F}$

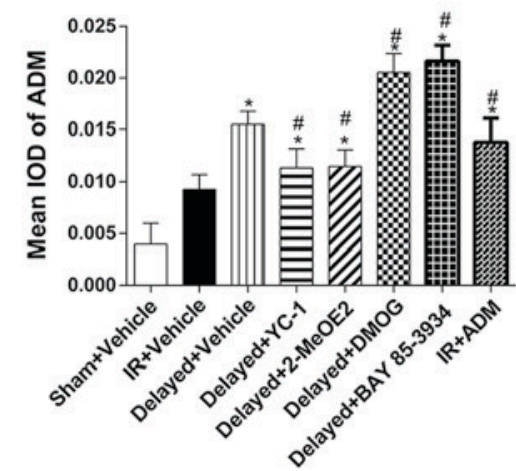

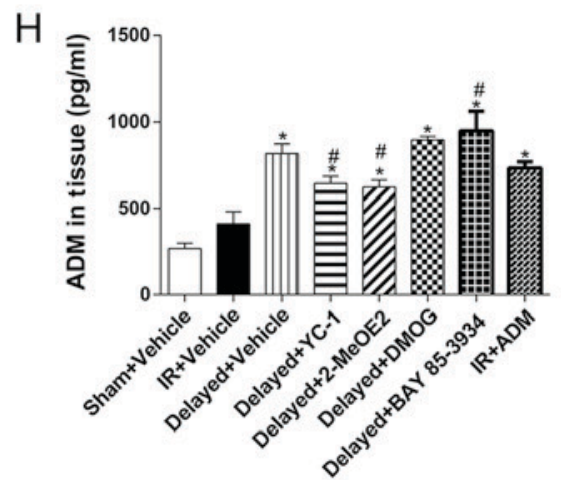

Figure 2. The expression of HIF-1 $\alpha$, ADM, and tubular score was influenced by accepting drugs (IHC; magnification, $\mathrm{x} 400$ ) ("P<0.05 vs. IR+Vehicle group; ${ }^{\#} \mathrm{P}<0.05$ vs. Delayed+Vehicle group). (A and B) The western blot analysis of HIF-1 $\alpha$ expression. (C and D) The immunohistochemistry staining of HIF-1 $\alpha$ in renal tissue. (E and F) The expression of ADM detected by immunohistochemistry. (G) The level of ADM in blood. (H) The level of ADM in renal tissue.

\section{Discussion}

RIPC is a less invasive method for reducing myocardial infarct size developed from ischemic preconditioning (IPC), offering similar protection by applying ischemia reperfusion to the organs or tissue far away from the protected target (3). RIPC was first and frequently applied during cardiac surgery in the clinic (2). However, it was rarely used in urology. Currently, 

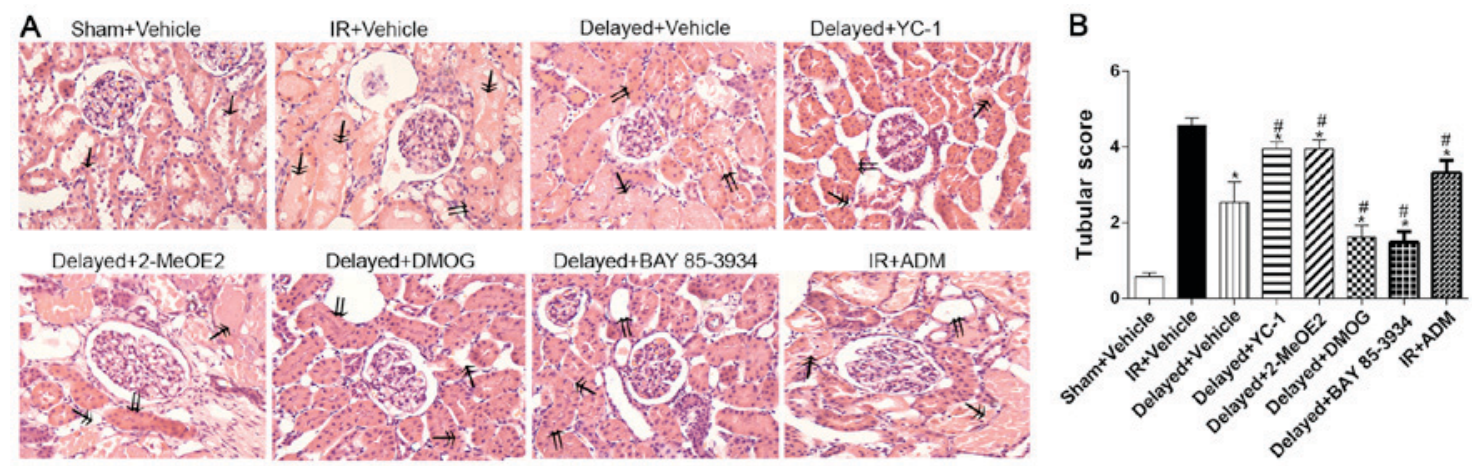

Figure 3. Renal tubular pathology ( $\mathrm{P}<0.05$ vs. IR+Vehicle group; ${ }^{*} \mathrm{P}<0.05$ vs. Delayed+Vehicle group; $\uparrow$ tubular epithelial cell flattening, brush border loss, and apoptosis; $\uparrow$ lumen flat expansion and cast; $\uparrow$ tubular lumen multifocal necrosis and debris). (A) Hematoxylin and eosin staining of renal tissue in different groups (HE; magnification, $\mathrm{x} 400)$. (B) The tubular score in renal tissue.

the protection offered by RIPC remains controversial $(3,5,6)$ and the mechanism is unclear. This was also one of the reasons we conducted the study.

Similar to IPC, the protective effect of RIPC had been demonstrated to have two phases or windows, with the first period of protection occurring and lasting 1 to $4 \mathrm{~h}$ immediately after precondition or acute RIPC, and subsequently the delayed RIPC appearing at $24 \mathrm{~h}$ and lasting $72 \mathrm{~h}$ (34). In part 1, the two phases of RIPC both could reduce the ischemia reperfusion damage and improve renal function indicators, and the delayed phase offered better protection that was adopted as RIPC protocol in part 2 (Table I, Fig. 1I).

The results of our experiment suggested HIF-1 $\alpha$ plays a key role in the renal protection offered by RIPC. HIF-1, consisting of an inducible $\alpha$ subunit and constitutive $\beta$ subunit, was a transcriptional factor whose expression was affected by oxygen concentration in the environment, regulating hundreds of downstream target gene expressions, including ADM (20). It played an important role in basic metabolism so that mice would die if the HIF-1 $\alpha$ gene was knocked down (35). HIF-1 also was involved in cancer metabolism and metastasis (36). First, the expression of HIF-1 $\alpha$ was significantly increased which was a positive correlation for renal protection. Second, in the Delayed+DMOG group receiving DMOG injection and the Delayed+BAY 85-3934 group receiving BAY 85-3934 injection, both of the two agents slowed the degradation of the HIF-1 $\alpha$, relatively increased the expression of the HIF-1, significantly improved the indicators of $\mathrm{Cr}$, BUN, MPO, MDA, lowered the tubular score, and further strengthened the protection of RIPC.

However, in the Delayed+YC-1 group and the Delayed+2-MeOE2, the expression of HIF-1 $\alpha$ was obviously inhibited and the expression of ADM in blood and tissue was decreased. In addition, the renal function indicators $\mathrm{Cr}$ and BUN, and oxidative damage indicators MPO, MDA, and tubular score in the Delayed+YC-1 group were worse than in the Delayed + Vehicle group. All the aforementioned findings suggested changing the expression of HIF-1 $\alpha$ could significantly strengthen or weaken the protective effects of RIPC.

ADM, a biologically actively peptide regulated by HIF-1, was first isolated from human pheochromocytoma tissue extracts (17). It has an important effect on heart and vascular protection, angiogenesis, central nervous system protection, tumor growth-promoting action, anti-inflammation, receptors, and intracellular signaling systems (18). Circulating ADM was rapidly metabolized with a half-life of about $20 \mathrm{~min}$, but exogenous ADM could increase the concentration in plasma and tissue (37). We found that when the expression of HIF-1 $\alpha$ increased or decreased, accordingly the expression of ADM also changed. High expression of HIF-1 $\alpha$ upregulated the concentration of ADM in blood and tissue. Elevated ADM might play an anti-inflammatory and antioxidant role, which could reduce the inflammatory infiltration and lipid peroxidation in the tissue suffering ischemia reperfusion, and provide protection to the microvasculature. However, the elevation of ADM was only significant in the delayed phase of RIPC. Furthermore, in the presence of exogenous ADM, the expression of HIF-1 $\alpha$ was also increased.

Thus, we inferred the protection in early or acute phase of RIPC was offered by the existent ADM, SOD, and other proteins that played an important role in antioxidation. Later in the delayed phase, the expression of HIF-1 $\alpha$ upregulated the transcription of these proteins, and the system could synthesize more antioxidants to fight against IRI. Furthermore, there might also be some positive feedback regulation mechanism between ADM and HIF-1 $\alpha$ that significantly increased the expression of ADM and thus help play a stronger role in the protection of renal function in the delayed phase rather than the acute phase. However, more research on how the positive feedback regulation mechanism worked is needed.

We found that ADM might play an important role in the protective effect of RIPC in renal function. After exogenous ADM was received, the concentration of ADM in blood was not significantly increased although it was obviously increased in renal tissue. Exogenous ADM improved the activity of SOD, lowered the activity of MPO, MDA, and improved tubular score. However, simply receiving ADM could not take place of the role of RIPC in renal protection.

In summary, RIPC could provide effective renal protection against ischemia reperfusion injury. The upregulated expression of HIF-1 $\alpha$ was the key to RIPC's protection. With the help of HIF-1, a series of antioxidant proteins was upregulated, which ultimately played a role in fighting against IRI. ADM was an important member of a series of downstream target proteins regulated by HIF-1, and it might form a positive 
feedback regulation pathway with HIF-1 $\alpha$, which made the delayed phase of RIPC exhibit stronger renal protection.

\section{Acknowledgements}

The present study was sponsored by the Natural Science Foundation of Shanghai (grant no. 14ZR1426900).

\section{References}

1. Murry CE, Jennings RB and Reimer KA: Preconditioning with ischemia: A delay of lethal cell injury in ischemic myocardium. Circulation 74: 1124-1136, 1986.

2. Przyklenk K, Bauer B, Ovize M, Kloner RA and Whittaker P: Regional ischemic 'preconditioning' protects remote virgin myocardium from subsequent sustained coronary occlusion Circulation 87: 893-899, 1993

3. Tapuria N, Kumar Y, Habib MM, Abu Amara M, Seifalian AM and Davidson BR: Remote ischemic preconditioning: A nove protective method from ischemia reperfusion injury-a review. J Surg Res 150: 304-330, 2008.

4. Wever KE, Warlé MC, Wagener FA, van der Hoorn JW, Masereeuw R, van der Vliet JA and Rongen GA: Remote ischaemic preconditioning by brief hind limb ischaemia protects against renal ischaemia-reperfusion injury: The role of adenosine. Nephrol Dial Transplant 26: 3108-3117, 2011.

5. Remote Preconditioning Trialists' Group, Healy DA, Khan WA Wong CS, Moloney MC, Grace PA, Coffey JC, Dunne C, Walsh SR, Sadat U, et al: Remote preconditioning and major clinical complications following adult cardiovascular surgery: Systematic review and meta-analysis. Int J Cardiol 176: 20-31, 2014.

6. Yang Y, Lang XB, Zhang P, Lv R, Wang YF and Chen JH: Remote ischemic preconditioning for prevention of acute kidney injury: A meta-analysis of randomized controlled trials. Am J Kidney Dis 64: 574-583, 2014

7. Hausenloy DJ and Yellon DM: Remote ischaemic preconditioning: Underlying mechanisms and clinical application. Cardiovasc Res 79: 377-386, 2008.

8. Saxena P, Newman MA, Shehatha JS, Redington AN and Konstantinov IE: Remote ischemic conditioning: Evolution of the concept, mechanisms, and clinical application. J Card Surg 25: 127-134, 2010

9. Pell TJ, Baxter GF, Yellon DM and Drew GM: Renal ischemia preconditions myocardium: Role of adenosine receptors and ATP-sensitive potassium channels. Am J Physiol 275: H1542-H1547, 1998

10. Schoemaker RG and van Heijningen CL: Bradykinin mediates cardiac preconditioning at a distance. Am J Physiol Heart Circ Physiol 278: H1571-H1576, 2000.

11. Patel HH, Moore J, Hsu AK and Gross GJ: Cardioprotection at a distance: Mesenteric artery occlusion protects the myocardium via an opioid sensitive mechanism. J Mol Cell Cardiol 34: 1317-1323, 2002

12. Tang ZL, Dai W, Li YJ and Deng HW: Involvement of capsaicin-sensitive sensory nerves in early and delayed cardioprotection induced by a brief ischaemia of the small intestine. Naunyn Schmiedebergs Arch Pharmacol 359: 243-247, 1999.

13. Kant R, Diwan V, Jaggi AS, Singh N and Singh D: Remote renal preconditioning-induced cardioprotection: A key role of hypoxia inducible factor-prolyl 4-hydroxylases. Mol Cell Biochem 312: 25-31, 2008.

14. Hill P, Shukla D, Tran MG, Aragones J, Cook HT, Carmeliet P and Maxwell PH: Inhibition of hypoxia inducible factor hydroxylases protects against renal ischemia-reperfusion injury. J Am Soc Nephrol 19: 39-46, 2008

15. Serejo FC, Rodrigues LF Jr, da Silva Tavares KC, de Carvalho AC and Nascimento JH: Cardioprotective properties of humoral factors released from rat hearts subject to ischemic preconditioning. J Cardiovasc Pharmacol 49: 214-220, 2007.

16. Shimizu M, Tropak M, Diaz RJ, Suto F, Surendra H, Kuzmin E, Li J, Gross G, Wilson GJ, Callahan J and Redington AN: Transient limb ischaemia remotely preconditions through a humoral mechanism acting directly on the myocardium: Evidence suggesting cross-species protection. Clin Sci (Lond) 117: 191-200, 2009.
17. Kitamura K, Kangawa K, Kawamoto M, Ichiki Y, Nakamura S, Matsuo H and Eto T: Adrenomedullin: A novel hypotensive peptide isolated from human pheochromocytoma. Biochem Biophys Res Commun 192: 553-560, 1993.

18. Kato J and Kitamura K: Bench-to-bedside pharmacology of adrenomedullin. Eur J Pharmacol 764: 140-148, 2015.

19. Kubo K, Tokashiki M, Kuwasako K, Tamura M, Tsuda S, Kubo S, Yoshizawa-Kumagaye K, Kato J and Kitamura K: Biological properties of adrenomedullin conjugated with polyethylene glycol. Peptides 57: 118-121, 2014.

20. Majmundar AJ, Wong WJ and Simon MC: Hypoxia-inducible factors and the response to hypoxic stress. Mol Cell 40: 294-309, 2010.

21. Ateş E, Genç E, Erkasap N, Erkasap S, Akman S, Firat P, Emre S and Kiper H: Renal protection by brief liver ischemia in rats. Transplantation 74: 1247-1251, 2002.

22. Oxman T, Arad M, Klein R, Avazov N and Rabinowitz B: Limb ischemia preconditions the heart against reperfusion tachyarrhythmia. Am J Physiol 273: H1707-H1712, 1997.

23. Zhang Z, Yan J, Taheri S, Liu KJ and Shi H: Hypoxia-inducible factor 1 contributes to $\mathrm{N}$-acetylcysteine's protection in stroke. Free Radic Biol Med 68: 8-21, 2014.

24. Schaible EV, Windschügl J, Bobkiewicz W, Kaburov Y, Dangel L, Krämer T, Huang C, Sebastiani A, Luh C, Werner C, et al: 2-Methoxyestradiol confers neuroprotection and inhibits a maladaptive HIF-1 $\alpha$ response after traumatic brain injury in mice. J Neurochem 129: 940-954, 2014.

25. Zhao X, Jin $\mathrm{Y}$, Li $\mathrm{H}$, Wang $\mathrm{Z}$, Zhang $\mathrm{W}$ and Feng $\mathrm{C}$ : Hypoxia-inducible factor 1 alpha contributes to pulmonary vascular dysfunction in lung ischemia-reperfusion injury. Int $\mathrm{J}$ Clin Exp Pathol 7: 3081-3088, 2014

26. Flamme I, Oehme F, Ellinghaus P, Jeske M, Keldenich J and Thuss U: Mimicking hypoxia to treat anemia: HIF-stabilizer BAY 85-3934 (Molidustat) stimulates erythropoietin production without hypertensive effects. PLoS One 9: e111838, 2014.

27. Dwivedi AJ, Wu R, Nguyen E, Higuchi S, Wang $\mathrm{H}$, Krishnasastry K, Marini CP, Ravikumar TS and Wang P: Adrenomedullin and adrenomedullin binding protein-1 prevent acute lung injury after gut ischemia-reperfusion. J Am Coll Surg 205: 284-293, 2007.

28. Yeo EJ, Chun YS, Cho YS, Kim J, Lee JC, Kim MS and Park JW: YC-1: A potential anticancer drug targeting hypoxia-inducible factor 1. J Natl Cancer Inst 95: 516-525, 2003.

29. Zudaire E, Martı́nez A and Cuttitta F: Adrenomedullin and cancer. Regul Pept 112: 175-183, 2003

30. Hu CJ, Wang LY, Chodosh LA, Keith B and Simon MC: Differential roles of hypoxia-inducible factor 1alpha (HIF-1alpha) and HIF-2alpha in hypoxic gene regulation. Mol Cell Biol 23: 9361-9374, 2003

31. Yuan Q, Bleiziffer O, Boos AM, Sun J, Brandl A, Beier JP, Arkudas A, Schmitz M, Kneser U and Horch RE: PHDs inhibitor DMOG promotes the vascularization process in the AV loop by HIF-1a up-regulation and the preliminary discussion on its kinetics in rat. BMC Biotechnol 14: 112, 2014.

32. Paller MS, Hoidal JR and Ferris TF: Oxygen free radicals in ischemic acute renal failure in the rat. J Clin Invest 74: 1156-1164, 1984.

33. Yurdakul T, Kulaksizoglu H, Piskin MM, Avunduk MC, Ertemli E, Gokçe G, Barişkaner H, Byükbaş S and Kocabas V: Combination antioxidant effect of $\alpha$-tocoferol and erdosteine in ischemia-reperfusion injury in rat model. Int Urol Nephrol 42: 647-655, 2010.

34. Loukogeorgakis SP, Panagiotidou AT, Broadhead MW, Donald A, Deanfield JE and MacAllister RJ: Remote ischemic preconditioning provides early and late protection against endothelial ischemia-reperfusion injury in humans: Role of the autonomic nervous system. J Am Coll Cardiol 46: 450-456, 2005.

35. Cai Z, Luo W, Zhan H and Semenza GL: Hypoxia-inducible factor 1 is required for remote ischemic preconditioning of the heart. Proc Natl Acad Sci USA 110: 17462-17467, 2013.

36. Tsai YP and Wu KJ: Hypoxia-regulated target genes implicated in tumor metastasis. J Biomed Sci 19: 102, 2012.

37. Kitamura K, Kangawa K, Kawamoto M, Ichiki Y, Nakamura S, Matsuo $\mathrm{H}$ and Eto T: Adrenomedullin: A novel hypotensive peptide isolated from human pheochromocytoma. 1993. Biochem Biophys Res Commun 425: 548-555, 2012.

This work is licensed under a Creative Commons Attribution-NonCommercial-NoDerivatives 4.0 International (CC BY-NC-ND 4.0) License. 